The Journal of Experimental Biology 215, 2369-2381 (C) 2012. Published by The Company of Biologists Ltd doi:10.1242/jeb.053744

RESEARCH ARTICLE

\title{
Flow structure and transport characteristics of feeding and exchange currents generated by upside-down Cassiopea jellyfish
}

\author{
Arvind Santhanakrishnan ${ }^{1, \star}$, Makani Dollinger ${ }^{2}$, Christina L. Hamlet ${ }^{3}$, Sean P. Colin ${ }^{4,5}$ and Laura A. Miller ${ }^{2}$ \\ ${ }^{1}$ Department of Biomedical Engineering, Georgia Institute of Technology, 315 Ferst Drive NW, Atlanta, GA 30332-0363, USA, \\ ${ }^{2}$ Department of Mathematics, University of North Carolina, CB 3250, Chapel Hill, NC 27599, USA, ${ }^{3}$ Department of Mathematics, \\ North Carolina State University, Raleigh, NC 27695, USA, ${ }^{4}$ Marine Biology and Environmental Science, Roger Williams University, \\ Bristol, RI 02809, USA and ${ }^{5}$ Whitman Center, Marine Biological Laboratories, Woods Hole, MA 02543, USA \\ *Author for correspondence (arvind7@gatech.edu)
}

\begin{abstract}
SUMMARY
Quantifying the flows generated by the pulsations of jellyfish bells is crucial for understanding the mechanics and efficiency of their swimming and feeding. Recent experimental and theoretical work has focused on the dynamics of vortices in the wakes of swimming jellyfish with relatively simple oral arms and tentacles. The significance of bell pulsations for generating feeding currents through elaborate oral arms and the consequences for particle capture are not as well understood. To isolate the generation of feeding currents from swimming, the pulsing kinematics and fluid flow around the benthic jellyfish Cassiopea spp. were investigated using a combination of videography, digital particle image velocimetry and direct numerical simulation. During the rapid contraction phase of the bell, fluid is pulled into a starting vortex ring that translates through the oral arms with peak velocities that can be of the order of $10 \mathrm{~cm} \mathrm{~s}^{-1}$. Strong shear flows are also generated across the top of the oral arms throughout the entire pulse cycle. A coherent train of vortex rings is not observed, unlike in the case of swimming oblate medusae such as Aurelia aurita. The phase-averaged flow generated by bell pulsations is similar to a vertical jet, with induced flow velocities averaged over the cycle of the order of $1-10 \mathrm{~mm} \mathrm{~s}^{-1}$. This introduces a strong near-horizontal entrainment of the fluid along the substrate and towards the oral arms. Continual flow along the substrate towards the jellyfish is reproduced by numerical simulations that model the oral arms as a porous Brinkman layer of finite thickness. This two-dimensional numerical model does not, however, capture the far-field flow above the medusa, suggesting that either the three-dimensionality or the complex structure of the oral arms helps to direct flow towards the central axis and up and away from the animal.
\end{abstract}

Supplementary material available online at http://jeb.biologists.org/cgi/content/full/215/14/2369/DC1

Key words: upside-down jellyfish, Cassiopea, flow field, feeding, currents, swimming.

Received 11 November 2010; Accepted 15 March 2012

\section{INTRODUCTION}

Medusae represent one of the earliest organisms to employ unsteady propulsion for locomotion and feeding (Costello et al., 2008). Recent work using digital particle image velocimetry (DPIV) and computational fluid dynamics has elucidated the mechanisms by which jellyfish effectively propel themselves and feed. The specific details on the relationship between unsteady fluid dynamics and swimming efficiency have been investigated using mathematical modeling (Daniel, 1983; Daniel, 1984; Dabiri et al., 2007), experiments (Costello and Colin, 1994; McHenry and Jed, 2003; Dabiri et al., 2005) and numerical simulations (Sahin et al., 2009; Lipinski and Mohseni, 2009). More recent studies have used DPIV and Lagrangian coherent structures (LCS) to characterize the vortex shedding and fluid mixing due to the pulsations of the bell (Dabiri et al., 2010; Peng and Dabiri, 2008a; Peng and Dabiri, 2008b; Peng and Dabiri, 2009; Lipinski and Mohseni, 2009). The vast majority of this experimental and computational work has focused on species with relatively simple morphologies, while the hydrodynamics of flow through and around elaborate oral arm structures that comprise a large portion of the body mass has not been rigorously explored. Furthermore, much of the previous work considered the problem of coupled swimming and feeding, such as in the case of Aurelia aurita (Castello and Colin, 1995; Peng and Dabiri, 2009). Differences in the resulting fluid dynamics for species with minimal and prominent oral arms should have implications for strategies of prey capture and nutrient exchange.

In this paper, we capitalized upon the unique features of Cassiopea spp. to characterize the feeding currents generated, uncoupled from swimming and through prominent oral arm structures. The upside-down jellyfish is found in shallow, protected and quiescent marine environments saturated with sunlight (Templeman and Kingsford, 2010; Welsh et al., 2009). Mature medusae rarely swim and instead rest their bells upside-down on the substrate, directing their oral arms towards the sunlight (Arai, 1997). Through periodic contractions and relaxations of the bell margin, Cassiopea spp. drive water into and away from the subumbrellar cavity and over the oral arms. This volume of entrained fluid is used to sample for particulate prey such as copepods and other zooplankton. The benthic lifestyle of Cassiopea spp. makes them dependent upon self-generated flows to bring nutrients and food to the animal. The generation of feeding currents is likely significant to these organisms as their habitats are typically characterized by low flow velocities. For example, maximum flow velocities near and within sea grass beds can be of the order of 


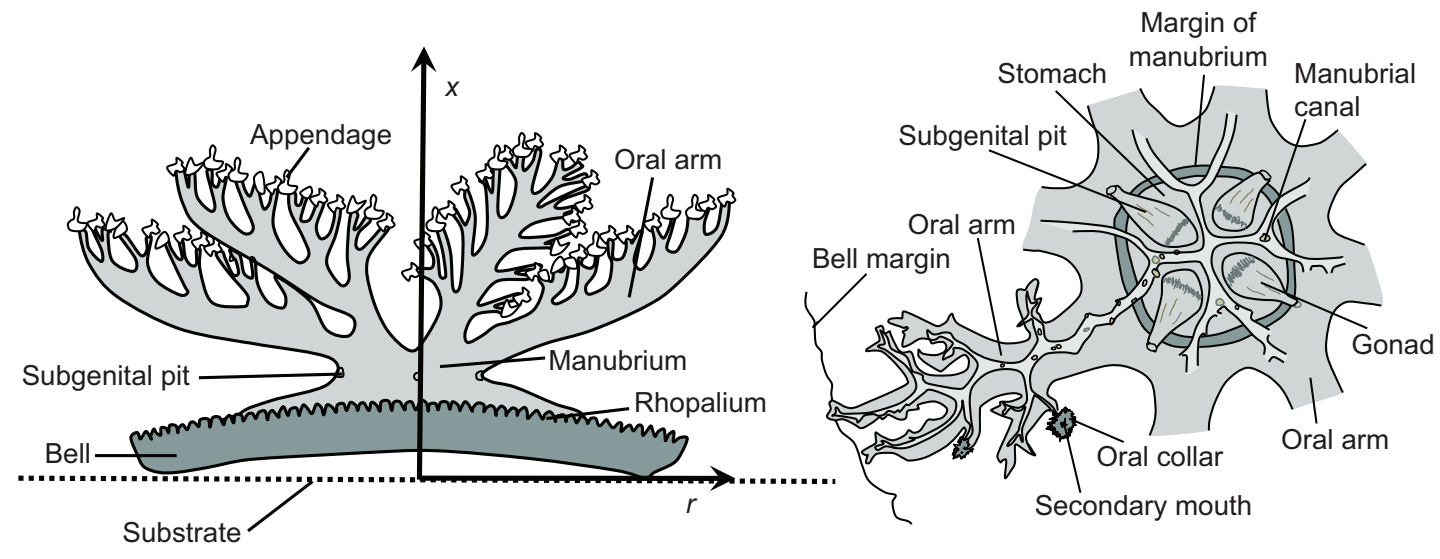

Fig. 1. Morphology of Cassiopea in side view (left) and top view (right) where the medusa is shown in its 'upside-down' orientation (modified from Hamlet et al., 2011) [redrawn from Hyman (Hyman, 1940)]. The axes used in the analysis are shown on the left. $x$ represents the axial or vertical position from the substrate; $r$ represents the radial or horizontal position measured from the center of the jellyfish. This coordinate system was used for bell kinematics and phase-averaged digital particle image velocimetry (DPIV) flow fields. For time-resolved DPIV measurements, the coordinate system was translated in the $r$ direction by $10 \mathrm{~mm}$. The manubrium is the central column of the jellyfish that emerges from the center of the oral surface of the bell with eight frilly oral arms radiating from it. Only one oral arm is drawn in its entirety from the top view. The data from organisms presented in this paper varied from 2 to $6 \mathrm{~cm}$ in bell diameter. There were also variations in the frilly branches of the oral arms among them.

$1 \mathrm{~cm} \mathrm{~s}^{-1}$ or lower (Bartleson, 2004). Flow rates within the boundary layer along the ocean floor are likely an order of magnitude lower than this. Maximum velocities within mangrove swamps may reach up to $0.7 \mathrm{~m} \mathrm{~s}^{-1}$, but flow rates near the sea floor are likely significantly lower (Wolanski, 1992).

Because Cassiopea belong to the Order Rhizostomeae, they do not possess tentacles or a primary mouth, in contrast to other commonly studied jellyfish such as $A$. aurita. In its place are multiple secondary mouths contained within the frilly oral arm branches. These differences in morphology may have implications for feeding performance and feeding strategies. The tentacles in A. aurita are positioned within the starting and stopping vortices generated during bell pulsations so as to maximize the possibility of capturing prey throughout the entire pulsing cycle (Dabiri et al., 2005). The prey captured in these strongly rotational regions of the fluid are also less likely to escape. The morphology of Cassiopea spp. and other Rhizostomeae is markedly different from this. Depending upon the species, the oral arms can extend to lengths greater than the maximum bell radius. The oral surface of Cassiopea consists of eight pinnately or irregularly branched appendages that are fused to the manubrium (Fig. 1). The presence of multiple secondary mouths throughout the oral arms suggests that fluid mixing should occur over the majority of the surface to maximize the rate of prey capture. A recent computational study motivated by the experiments presented in this paper supports this conjecture, and the results suggest that the presence of prominent oral arms can significantly alter the bulk flow of fluid (Hamlet et al., 2011).

In this paper, we present quantitative measurements of the pulsing kinematics and the resultant fluid dynamics of the currents generated by Cassiopea spp. DPIV was used to characterize the time-resolved and phase-averaged large-scale flow fields around the bell. The time-resolved measurements were conducted using highspeed video at 1000 frames s$^{-1}$ to investigate the formation of flow structures during the different stages of the pulse cycle. The phaseaveraged measurements were conducted at 30 frames s$^{-1}$ to characterize the overall transport of fluid around the organism. The results were then compared with direct numerical simulations that model the oral arms as a porous Brinkman layer. The dependence of the fluid dynamics on the morphology of oblate medusae with prominent oral arms as well as the implications of the results toward transport processes in Cassiopea spp. medusae are discussed.

\section{MATERIALS AND METHODS Specimen collection and handling}

Cassiopea frondosa (Pallas 1774) and Cassiopea xamachana Bigelow 1892 were obtained from Gulf Specimen Marine Lab, FL, USA, during July 2009, Carolina Biological Supply, NC, USA, during September 2009, and the New England Aquarium, Boston, MA, USA, during summer 2009. The organisms were transported overnight to the laboratory and placed in a 291 glass aquarium maintained at a temperature of around $20-22^{\circ} \mathrm{C}$. The experimental data were acquired from five individuals that ranged from 2 to $6 \mathrm{~cm}$ in bell diameter, defined during the stage of full bell relaxation. For the purposes of observation, an individual medusa was transferred from the holding tank to a smaller 101 glass aquarium that was $20.7 \times 40.3 \mathrm{~cm}$ at the base and $26 \mathrm{~cm}$ in height. Black sand was used in the observation tank to reduce glare in the optical measurements. Individuals placed in the observation tank took between 5 and 20 min to settle to the sandy bottom in their natural 'upside-down' orientation.

The high-speed video recording was performed at the Marine Biological Laboratories, Woods Hole, MA, USA. Cassiopea medusae were maintained in 201 aquaria at $22^{\circ} \mathrm{C}$. To collect video recordings, individuals with bell diameters of about $2 \mathrm{~cm}$ were placed into a glass filming vessel that was $15 \times 40 \mathrm{~cm}$ at the base and $30 \mathrm{~cm}$ in height.

\section{Bell kinematics}

Medusae were filmed using a Canon XH A1 camcorder at a rate of 30 frames s$^{-1}$. Standard room lighting was used for most video captures and an additional backlight was employed in certain cases to obtain better resolution. A centimeter grid was used behind the aquarium for scale reference. The focal planes of the videos were adjusted to align with the plane passing through the center of the bell and parallel to the front and back walls of the aquarium. The videos were digitized using the DLTdv3 program (Hedrick, 2008) in Matlab. From each frame, the instantaneous bell profile was extracted. The instantaneous fineness ratio $F_{\text {i }}$, defined as the ratio 
of the bell height $(h)$ to the diameter ( $d$ ) at any particular time, was estimated from the digitized images. For quantifying the bell profiles, radial symmetry about the oral-aboral axis was assumed and only one-half of the bell motion was digitized. It must be noted that because the medusae were resting on the bottom of the tank, only the regions of the bell that noticeably moved during a pulsing cycle were digitized. To spatially describe the bell curvature, nine points were selected per frame starting from the bell margin to the resting point of the bell on the substrate. These nine points were tracked across $14-15$ frames per pulsing cycle.

\section{DPIV}

Time-resolved, high-speed DPIV measurements were acquired on a medusa of bell diameter $(d)$ equal to $2 \mathrm{~cm}$ and at a camera frame rate of $1000 \mathrm{~Hz}$. Time-resolved measurements were also taken from five other specimens at a rate of $30 \mathrm{~Hz}$. For the high-speed measurements, a CMOS camera (Fastcam 1024 PCI, Photron, San Diego, CA, USA) with a $1024 \times 1024$ pixel array was used for capturing images. The flow field was illuminated from a $2 \mathrm{~W}$ continuous red laser, which emitted light at a wavelength of $680 \mathrm{~nm}$. The laser beam was converted into a planar sheet $\sim 1 \mathrm{~mm}$ thick using a set of focusing optics. The laser sheet was oriented perpendicular to the substrate and through the center of the medusa, as shown in Fig. 2A. For each DPIV run, 4315 individual images were recorded and processed to generate two-dimensional velocity fields. The velocities of particles illuminated in the laser sheet were determined from sequential images analyzed using a cross-correlation algorithm (LaVision, Ypsilanti, MI, USA). Image pairs were analyzed with shifting overlapping interrogation windows of decreasing size $(64 \times 64$ pixels then $32 \times 32$ pixels $)$.

To quantify the average bulk flow generated by the bell pulsations of Cassiopea, phase-averaged data were taken on a medusa of bell diameter $(d) 6 \mathrm{~cm}$. To seed the water for the purpose of tracking the flow, small volume concentrations of Artemia spp. eggs and some hatched nauplii (1-2 days old, around $0.5 \mathrm{~mm}$ in size) were added to the tank. Because all data were averaged over an order of 100 cycles, individual movements of nauplii or other zooplankton did not significantly affect the results. The flow field was illuminated via a $50 \mathrm{~mJ}$ double-pulsed neodymium-doped yttrium aluminium garnet (Nd:YAG) laser (Continuum, Santa Clara, CA, USA), which emitted light at a wavelength of $532 \mathrm{~nm}$ with a maximum repetition rate of $15 \mathrm{~Hz}$. The laser beam was converted into a planar sheet $\sim 3 \mathrm{~mm}$ thick using a set of focusing optics. A 14-bit charge-coupled device (CCD) camera (Imager Pro X 2M, LaVision) with a $1600 \times 1200$ pixel array was used for capturing images at a camera frame rate of $30 \mathrm{~Hz}$. The time interval of separation between two images in an image pair was adjusted to be in the range $0.01-0.03 \mathrm{~s}$. The maximum displacement of the seed particles was always maintained at below 4 pixels. For each DPIV run, 340 individual images were recorded for processing, resulting in a minimum of 170 velocity vector fields from which to generate two-dimensional velocity fields. Each instantaneous DPIV realization consisted of an array of $100 \times 75$ vectors, with each vector being separated by a width of 12 pixels.

For both the time-resolved and phase-averaged measurements, the laser sheet was oriented to pass through the oral-aboral axis of the organism and parallel to the front and back walls of the aquarium. Shadows formed after the laser sheet intersected the opaque Cassiopea medusa. As a result, only half of the phase-averaged flow field on one side of the oral-aboral axis will be presented. For processing the raw images, a single-pass fast Fourier transform (FFT)-based cross-correlation algorithm was used (Davis 7.0, LaVision). The interrogation window size used in the cross-
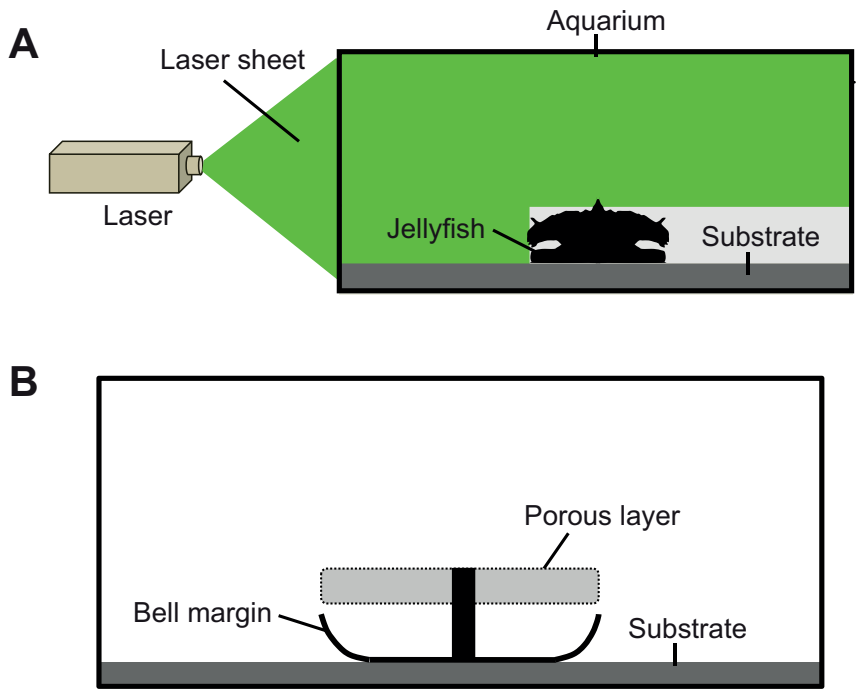

Fig. 2. Schematic diagrams (not to scale) for the experimental and corresponding numerical setup. (A) The upside-down jellyfish was placed in a standard aquarium. The flow field was illuminated by a planar laser sheet oriented perpendicular to the substrate and through the center of the medusa. (B) A numerical model of a jellyfish was constructed by modeling the bell as series of moving immersed boundary points, the porous oral arms as a stationary Brinkman layer, and the substrate and manubrium as stationary porous materials with porosity chosen such that flow through these pieces was negligible.

correlation analysis was $64 \times 64$ pixels for the instantaneous data and $32 \times 32$ pixels for the time-averaged data with $50 \%$ overlap. No preprocessing of the raw data was performed prior to this step. Postprocessing was performed in Matlab. No smoothing algorithms or other post-processing techniques were used on the data.

\section{Direct numerical simulation}

The immersed boundary method was used to solve the twodimensional (not axisymmetric) fluid-structure interaction problem of a pulsing Cassiopea medusa in a viscous, incompressible fluid. The model and method are as described elsewhere (Hamlet et al., 2011) with two modifications: (1) the mathematical models for the relaxed and contracted bell profiles were obtained by fitting the experimental data with third-order polynomials using a leastsquares regression, and (2) the porous layer representing the oral arms was represented as a Brinkman layer with finite thickness. The immersed boundary method (Peskin, 2002) and the exact numerical discretization used for these simulations (Peskin and McQueen, 1996) are as described previously.

Infinitely thin porous boundaries derived using Darcy's law have been used before in immersed boundary simulations to model parachutes (Kim and Peskin, 2006), jellyfish oral arms (Hamlet et al., 2011) and other porous structures (Stockie, 2009). The Brinkman model (Brinkman, 1947) was used in this study to model the oral arms as a porous layer with finite thickness. The Brinkman equations are a generalization of Darcy's law and are given as:

$$
\begin{gathered}
\rho\left(\mathbf{u}_{t}(\mathbf{x}, t)+\mathbf{u}(\mathbf{x}, t) \cdot \nabla \mathbf{u}(\mathbf{x}, t)\right)= \\
-\nabla p(\mathbf{x}, t)+\mu \Delta \mathbf{u}(\mathbf{x}, t)-\alpha^{2}(\mathbf{x}) \mu \mathbf{u}(\mathbf{x}, t)+\mathbf{f}(\mathbf{x}, t), \\
\nabla \cdot \mathbf{u}(\mathbf{x}, t)=0,
\end{gathered}
$$

where $\rho$ is the density of the fluid, $\mu$ is the dynamic viscosity, $\mathbf{u}(\mathbf{x}, t)$ is the fluid velocity, $p(\mathbf{x}, t)$ is the pressure and $\mathbf{f}(\mathbf{x}, t)$ is an 
external force per unit volume acting on the fluid. The independent variables are the position vector $\mathbf{x}$ and the time $t . \alpha^{2}$ is the reciprocal of the hydraulic permeability of the porous medium. It indicates the extent to which the flow is obstructed by the porous layer and may vary in space. Note that the Brinkman model is essentially the Navier-Stokes equations with the additional friction term $\alpha^{2}(\mathbf{x}) \mu \mathbf{u}(\mathbf{x}, t)$ as a result of the presence of the porous layer.

Regions with porous structures were modeled with positive values of $\alpha^{2}$ while open regions of the fluid domain had $\alpha^{2}$ set to zero. This was done by constructing porous 'clumps' using hyperbolic tangents as described elsewhere (Leiderman et al., 2008). For all simulations shown, $\alpha^{2}$ was set equal to $4.5 \times 10^{6} \mathrm{~m}^{-2}$ in the $x$ direction and $4.5 \times 10^{7} \mathrm{~m}^{-2}$ in the $y$-direction. This had the effect of directing the flow to move vertically through the layer in a manner similar to the infinitely thin model described by Hamlet and colleagues (Hamlet et al., 2011). $\alpha^{2}$ was varied from 0 to $4.5 \times 10^{10} \mathrm{~m}^{-2}$ to determine the range of $\alpha^{2}$ for which the flow was affected by the presence of the layer but not totally blocked by it $\left(1 \times 10^{5} \mathrm{~m}^{-2} \leq \alpha^{2} \leq 4.5 \times 10^{8} \mathrm{~m}^{-2}\right)$. Within this range the pattern of flow was similar, supporting the results reported by Hamlet and colleagues.

Fig. 2B shows the basic numerical setup. The Navier-Stokes equations were solved on a $630 \times 630$ periodic domain. Tethered immersed boundary points were used to construct walls along the edges of the domain to break the periodicity. A two-dimensional medusa was constructed by taking a slice through the central axis, similar to the cut the laser sheet makes in the experiments. The aboral surface of the bell was constructed using a line drawn along the substrate. The expanded, half-contracted, contracted and halfexpanded states of the bell margins were constructed using cubic polynomials. The bell was moved between the expanded and contracted states with a linear interpolation. To model structures of finite thickness, the substrate, oral arms and manubrium were constructed as porous layers. In the regions of the substrate and manubrium, $\alpha^{2}$ was set to $4.5 \times 10^{9} \mathrm{~m}^{-2}$. This corresponds to a permeability of nearly zero $\left(1 / \alpha^{2}=2.2 \times 10^{-10} \mathrm{~m}^{-2}\right)$ such that the regions acted like solid layers. Four pulse cycles were simulated for each case. Physical dimensions for the simulations are given in Table 1 .

\section{RESULTS}

\section{Kinematic observations}

The pulsing cycle of Cassiopea consists of a power stroke where the bell undergoes full contraction, followed by a recovery stroke that is a phase of full bell relaxation. In general, the average frequency at which the pulsing occurred was in the range $0.9 \pm 0.1 \mathrm{~Hz}$. Variations from this cycle duration were observed during target feeding of Artemia spp. nauplii to a medusa. In

Table 1. Physical dimensions for the numerical simulations of a $2 \mathrm{~cm}$ and $6 \mathrm{~cm}$ jellyfish

\begin{tabular}{lcc}
\hline Parameter & $2 \mathrm{~cm} \mathrm{model}$ & $6 \mathrm{~cm} \mathrm{model}$ \\
\hline Bottom of bell & $2 \mathrm{~cm}$ & $6 \mathrm{~cm}$ \\
Length of computational domain & $10 \times 10 \mathrm{~cm}$ & $30 \times 30 \mathrm{~cm}$ \\
Height of bottom of oral arms & $0.45 \mathrm{~cm}$ & $1.35 \mathrm{~cm}$ \\
Height of top of oral arms & $0.95 \mathrm{~cm}$ & $2.85 \mathrm{~cm}$ \\
Width of oral arms & $3.5 \mathrm{~cm}$ & $10.5 \mathrm{~cm}$ \\
Duration of contraction & $0.393 \mathrm{~s}$ & $0.44 \mathrm{~s}$ \\
Length of first pause & $0.01394 \mathrm{~s}$ & $0.0095 \mathrm{~s}$ \\
Length of expansion & $1.1214 \mathrm{~s}$ & $0.638 \mathrm{~s}$ \\
Length of second pause & $0.0737 \mathrm{~s}$ & $0.0124 \mathrm{~s}$ \\
Length of cycle & $1.602 \mathrm{~s}$ & $1.1 \mathrm{~s}$ \\
\hline
\end{tabular}

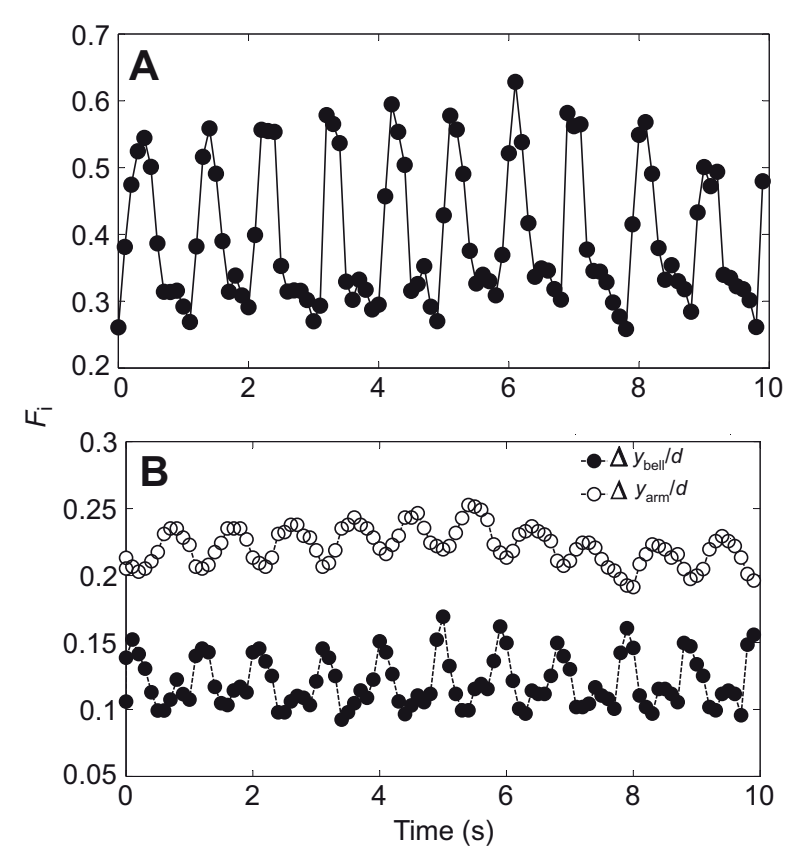

Fig. 3. (A) The instantaneous fineness ratio $\left(F_{\mathrm{i}}\right)$ vs time. $F_{\mathrm{i}}$ is calculated as the ratio of the height of the bell to the diameter. (B) The axial position of the tip of the bell (filled circles) and the tip of the oral arms (open circles) divided by the bell diameter. Data were taken from an individual Cassiopea of $9 \mathrm{~cm}$ diameter $(d)$. Profiles show variation in time over 10 pulsing cycles. Each cycle consists of at least 10 data points.

addition, certain environmental conditions also appeared to marginally affect the pulsing cycle, including the presence or absence of baseline currents in the aquarium, proximity to neighboring medusae and ambient light intensity. All measurements reported refer to non-swimming Cassiopea medusae in conditions of no baseline fluid flow.

The relative durations of bell motion between the power and recovery phases of the pulsing cycle were different (Fig. 3A). Note that $x$ describes the axial or vertical position measured from the surface, and $r$ is the radial or horizontal position measured from the center of the bell. Such asymmetries have been observed in other Scyphomedusae (Costello and Colin, 1994). The bell motion in Cassiopea was chiefly restricted to around the bell margin as a result of both the presence of a higher relative mesoglea content and their upside-down orientation. From the kinematic analyses, the position of the bell margin was tracked as a function of both the spatial location and time. The center of the bell was used as the reference point for tracking the position of the bell margin, and only one-half of the bell diameter was analyzed, assuming radial symmetry. The axial or vertical position of the tip of the oral arm and bell are shown in Fig. 3B as functions of time. The oral appendages of Cassiopea medusae used in this study typically extended to more than the bell radius. Near the end of the power stroke, the oral arms were oriented slightly in an upward direction to possibly increase the encounter rate of particulate matter within the local fluid entrained by the medusa.

The power stroke was $\sim 40 \%$ of the period of the pulsing cycle $(0.4 \pm 0.07 \mathrm{~s}$ compared with a $1.1 \pm 0.1 \mathrm{~s}$ period, averaged over 10 cycles) for a medusa of $9 \mathrm{~cm}$ bell diameter. During the contraction phase, the bell diameter decreased resulting in an increase in the fineness ratio (Fig.3A). During relaxation, the bell diameter increased and thus the fineness ratio decreased. The bell height 


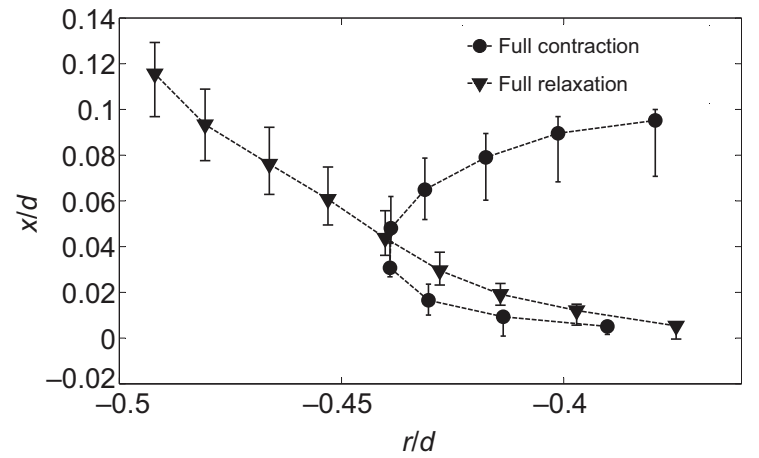

Fig. 4. Bell shape profiles for full relaxation and full contraction phases of the Cassiopea medusa pulsing cycle. The profiles were obtained from a medusa of $9 \mathrm{~cm}$ bell diameter and ensemble averaged over 9 pulsing cycles. $x$ and $r$ as in Fig. 1. The error bars represent the standard deviations of the bell motion across the 9 pulsing cycles considered.

remained constant for most of the recovery stroke, followed by a sharp increase during the power stroke. This finding is consistent with the kinematics of the free-swimming oblate medusae $A$. aurita reported elsewhere (Costello and Colin, 1994).
Compared with Aurelia (Bajcar et al., 2009), the tip of the bell margin in the fully contracted state of Cassiopea was moved further inward and closer to the axis of radial symmetry (Fig.4). It is important to note that during the end of the power stroke, the bell was pushed downward against the floor of the tank (Fig. 4). This is not seen in oblate medusan swimmers such as Aurelia and primarily arises from the difference in the preferred orientation of Cassiopea and the presence of the floor.

\section{Instantaneous flow measurements}

Instantaneous time-resolved DPIV realizations of the flow induced by a Cassiopea medusa of $2 \mathrm{~cm}$ bell diameter during contraction were visualized from velocity vector fields (Fig. 5). The pulsing cycle comprises (in ascending chronological order): a power stroke, which lasted for $24.53 \%$ of the cycle, a short pause, which lasted for $0.87 \%$ of the cycle, and a recovery stroke, which lasted for the remaining $74.6 \%$ of the cycle. To understand the initiation and development of the flow during the power stroke, specific times in each portion of the cycle are shown. For instance, 33\% of the power stroke in this case corresponds to roughly $8 \%$ of the overall pulsing cycle. At the start of the power stroke (Fig. 5A), the flow near the medusa at full bell relaxation is directed outward and
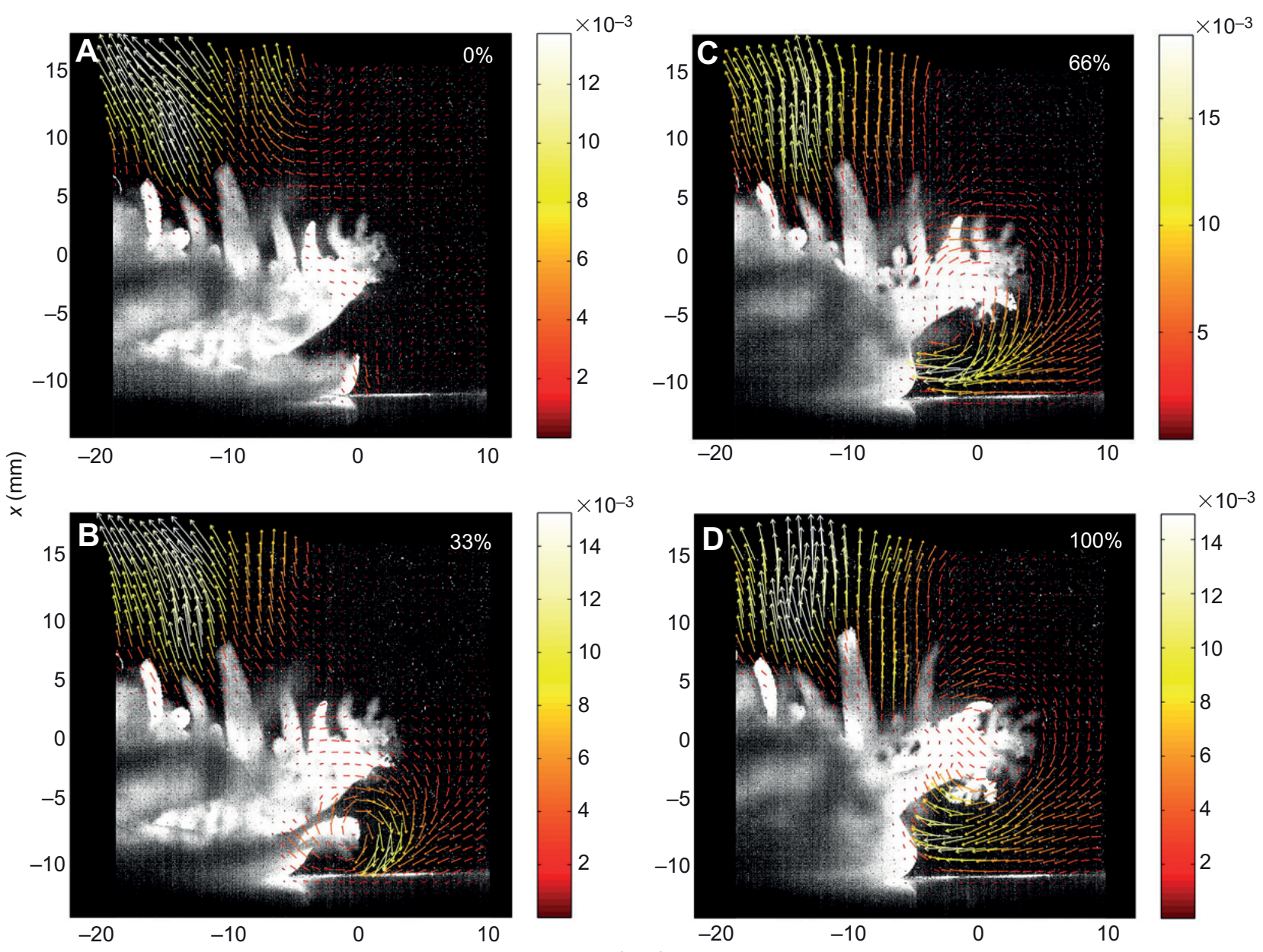

$r(\mathrm{~mm})$

Fig. 5. Time-resolved velocity vector fields of the flow induced by a Cassiopea medusa of $2 \mathrm{~cm}$ bell diameter. The instantaneous realizations shown correspond to four stages occurring during the power stroke portion of the pulsing cycle. The specific time intervals chosen within the power stroke are (A) $0 \%$ of the power stroke corresponding to the start cycle, (B) $33 \%$ of the power stroke, (C) $66 \%$ of the power stroke and (D) $100 \%$ of the power stroke. The lengths of the vectors indicate their relative magnitude and their direction indicates the direction of the flow. Velocity vector lengths are rescaled for each image, but the exact magnitudes are given by each color bar. The raw images were acquired at a frame rate of $1000 \mathrm{~Hz}$ to provide $1400-1600 \mathrm{images}$ per medusa pulsing cycle. 


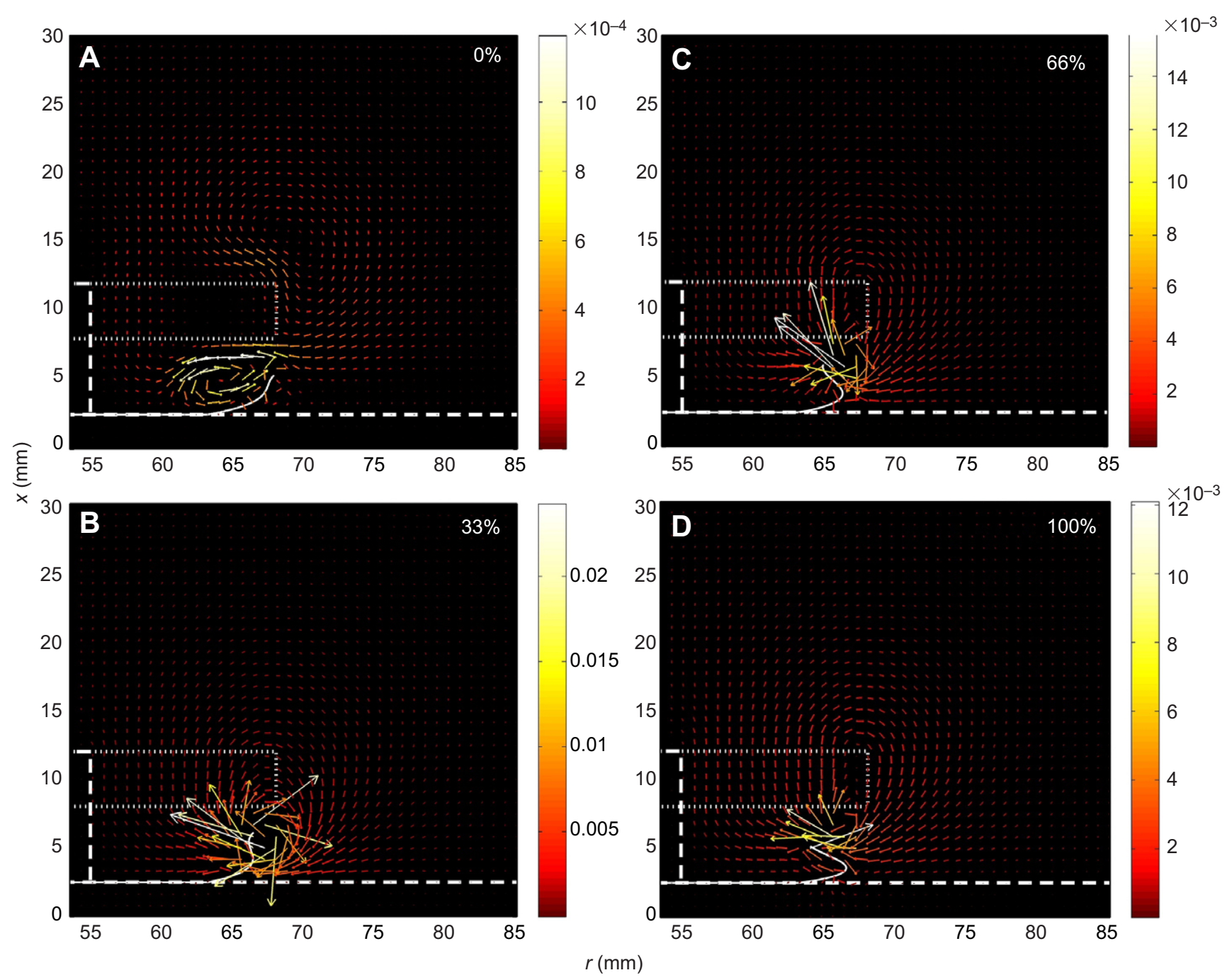

Fig. 6. Time-resolved velocity vector fields from numerical simulations for a model medusa of $2 \mathrm{~cm}$ bell diameter. The instantaneous realizations correspond to the same four stages during the power stroke shown in Fig. 5 , with A showing the start of the power stroke (0\%) and D the end of the power stroke $(100 \%)$. The borders of the substrate and manubrium are indicated by white dashed lines. The border of the porous arms is shown by the white dotted line. The bell margin is shown by a white line. The lengths of the vectors indicate their relative magnitude and their direction indicates the direction of the flow. Note that the relative vector lengths and color bar are rescaled in each image because of the rapid changes in the peak flow rates.

away from the bell margin; the flow above the medusa at this stage is primarily directed away from the oral surface in the form of a jet. At $33 \%$ of the power stroke (Fig. 5B), the bell margin is pushed outward from the fully relaxed state. This drives the flow adjacent to the tip of the bell margin away from the bell. Because of the presence of a boundary nearby, this fluid volume is forced to recirculate, thereby initiating the formation of a starting vortex that is centered on the tip of the bell margin. Further contraction of the bell at $66 \%$ of the power stroke moves the starting vortex along with the bell. Because of the short time scale and fast contraction speeds experienced during the power stroke, the bell motion occurring at $66 \%$ of the power stroke adds circulation strength to the starting vortex (Fig. 5C). The rotational nature of the starting vortex helps in entraining fluid from the region surrounding the medusa bell (Fig. 5D). A movie of the timeresolved flow fields for a medusa with a bell diameter of $4 \mathrm{~cm}$ is shown in supplementary material Movie 1.

Corresponding results from the numerical simulations are shown in Fig. 6. Similar to the experimental measurements, a strong starting vortex centered on the tip of the bell margin is formed and moves along with the bell (Fig. 6A,B). The numerical simulations allow the resolution of the flow near the boundary and within the bell, revealing the strong flow within the subumbrellar cavity (Fig. 6C). Peak flow rates are of the order of $2.75 \mathrm{~cm} \mathrm{~s}^{-1}$. Slower flow moves continuously along the substrate towards the bell and is then pushed upward through the oral arms (Fig. 6D). A movie of the instantaneous velocity field and vorticity for the simulation corresponding to a medusa with a bell diameter of $2 \mathrm{~cm}$ is shown in supplementary material Movie 2.

Instantaneous time-resolved DPIV realizations of the flow induced by a Cassiopea medusa of $2 \mathrm{~cm}$ bell diameter during bell expansion are shown in Fig. 7. The start of the recovery stroke was defined using the first instant when the bell starts to expand (Fig. 7A). At this time the starting vortex advects upward and radially outward from the medusa. At $20 \%$ of the recovery stroke, the expansion of the bell pushes the nearby fluid outward, creating a region of flow opposing the direction of the starting vortex (Fig. 7B). The bell relaxation at $40 \%$ of the recovery stroke continues to direct fluid 

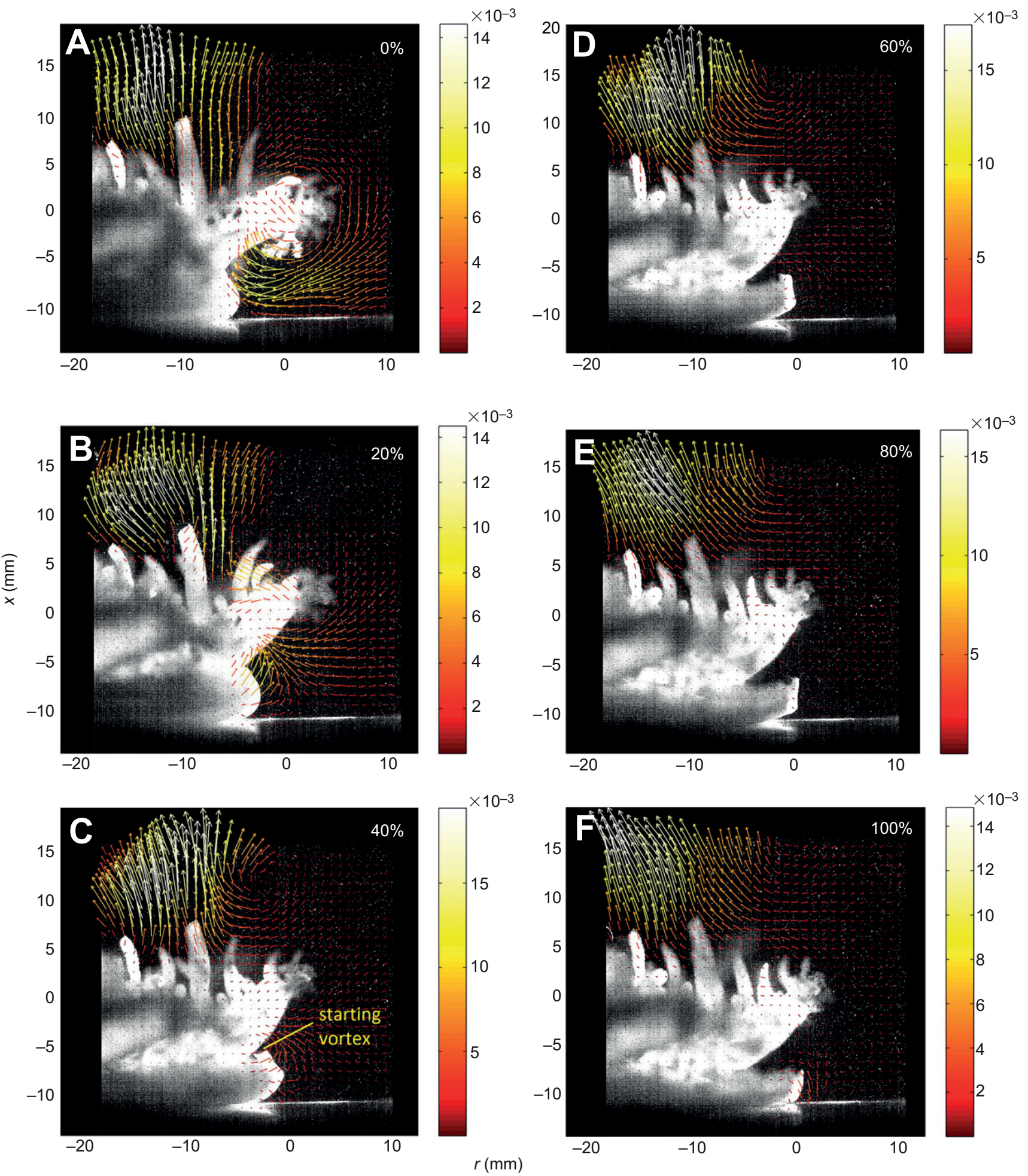

Fig. 7. Time-resolved velocity vector fields of the flow induced by a Cassiopea medusa of $2 \mathrm{~cm}$ bell diameter during bell expansion. The instantaneous realizations shown correspond to six stages $(A-F)$ occurring during the recovery stroke portion of the pulsing cycle: $0 \%$ indicates the start $(A)$ and $100 \%$ indicates the end of the recovery stroke $(F)$.

outward from the tip of the bell margin (Fig. 7C). The starting vortex has now advected above the surface of the oral arms, as seen by an increase in jet velocity (proportional to the vector lengths) above $x=5 \mathrm{~mm}$. At $60 \%$ of the recovery stroke, a stopping vortex is visible that is positively signed (counter-clockwise circulation) and centered on the tip of the bell margin (Fig. 7D). This flow structure rotates counter to the starting vortex and plays an important role in entraining slow-moving fluid from near the floor into the subumbrellar region. The flow within the stopping vortex slows upon further bell relaxation at $80 \%$ (Fig. 7E). At full bell relaxation, the bell margin is fully expanded and flow entrained in the subumbrellar cavity continues to be expelled outward (Fig. 7F). 

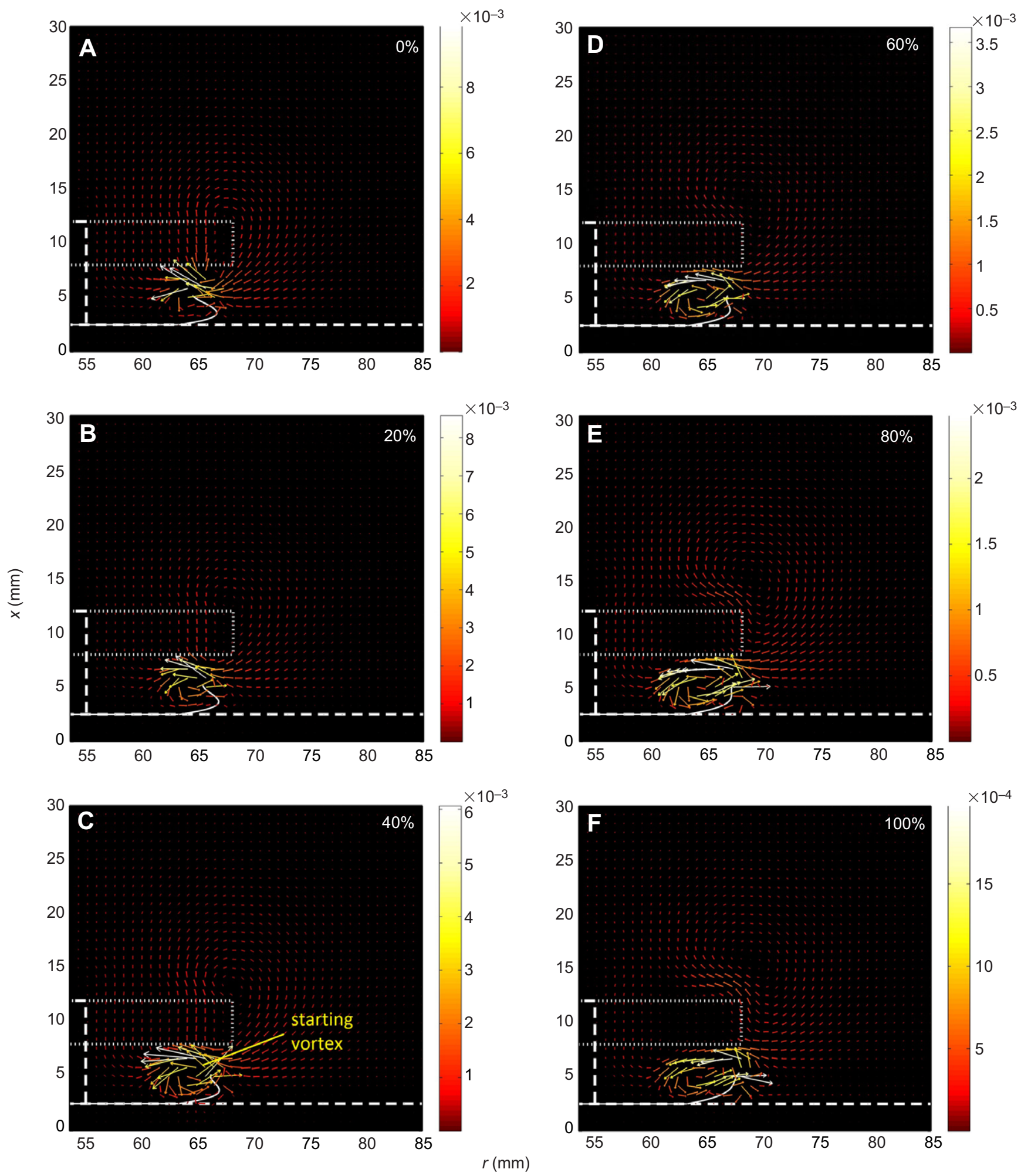

Fig. 8. Time-resolved velocity vector fields from numerical simulations for a model medusa of $2 \mathrm{~cm}$ bell diameter. The instantaneous realizations shown correspond to six stages (A-F) occurring during the recovery stroke portion of the pulsing cycle: $0 \%$ indicates the start (A) and $100 \%$ indicates the end of the recovery stroke $(\mathrm{F})$. The borders of the substrate and manubrium are given by white dashed lines. The border of the porous arms is given by the white dotted line. The bell margin is shown by a white line. Note that the relative vector lengths and color bar are rescaled in each image because of the rapid changes in the peak flow rates.

Corresponding results from the numerical simulations are shown in Fig. 8. During bell expansion, the counter-rotating stopping vortex is clearly visible within the bell margin (Fig. 8B-F). The placement of the oppositely spinning starting and stopping vortices continues to pull fluid into the bell from along the substrate during expansion (see supplementary material Movie2). Peak flow rates are of the order of $1 \mathrm{~cm} \mathrm{~s}^{-1}$ within the stopping vortex. Similar to the experimental findings, low flow rates are observed near the vortex 

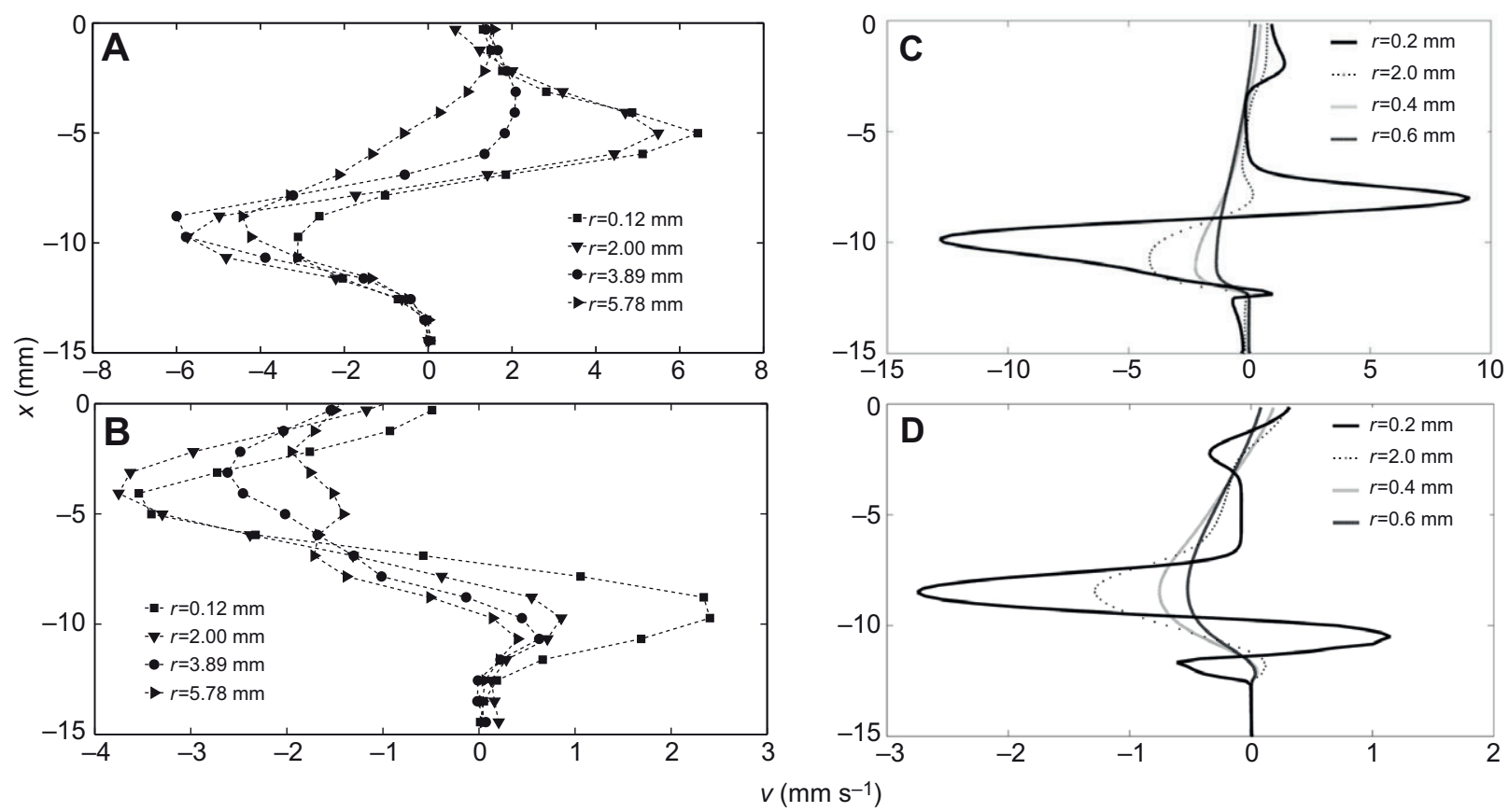

Fig. 9. Axial distribution of the radial (horizontal direction) component of the time-resolved flow velocity ( $v$ ) generated by a Cassiopea medusa (A,B) and a numerical model $(C, D)$ of $2 \mathrm{~cm}$ bell diameter. Velocities are shown for four positions of varying distance from the center of the bell $(r=-10 \mathrm{~mm})$. (A,C) $33 \%$ of the power stroke; (B,D) $40 \%$ of the recovery stroke.

core. Throughout the expansion, flow continues upwards through the oral arms. The numerical simulations also reveal a strong jet of flow during bell expansion moving towards the central axis between the bell margin and the oral arms (Fig. 8B-D). This jet was observed in the experiments but could not be resolved using DPIV because of the opacity of the organisms.

The magnitude of the flow generated by the starting and stopping vortices for both the DPIV measurements and the numerical simulations can be examined from the radial velocity profiles shown in Fig. 9. For the experimental measurements at 33\% of the power stroke, the radial velocity at $r=0.12 \mathrm{~mm}$ changes in sign at $x=-8 \mathrm{~mm}$, which is roughly the coordinates of the tip of the bell margin at that instant. Similar patterns of fluid motion are also observed in the numerical simulations. The outward motion of the bell during the power stroke initiates the starting vortex formation, and the peak radial velocity values (positive when the flow is moving away from the medusa) reach about $7 \mathrm{~mm} \mathrm{~s}^{-1}$ in experiments and $12 \mathrm{~mm} \mathrm{~s}^{-1}$ in simulations. Peak values for the larger medusa (see supplementary material Fig. S1) may reach up to $7-8 \mathrm{~cm} \mathrm{~s}^{-1}$. With increasing radial distance from the medusa, the positively signed radial velocity tends to decrease above the oral surface. The negatively signed radial velocity, indicative of flow moving toward the bell, peaks at about $r=4 \mathrm{~mm}$ in the experiments and is lowest at the tip of the bell $(r=0.12 \mathrm{~mm})$ where the starting vortex core is located. Similar flow patterns are also observed in numerical simulations.

Peak flow velocities during the recovery stroke occur near $40 \%$ of this phase (Fig. 9B,D). This corresponds to the formation of the stopping vortex. Note that these values are lower than those measured during the formation of the starting vortex. The velocity of the flow expelled out of the medusa peaks at around $2 \mathrm{~mm} \mathrm{~s}^{-1}$ in the experiments and at about $1 \mathrm{~mm} \mathrm{~s}^{-1}$ for the numerical simulations. In both cases, the velocities decrease as one moves radially outward. The asymmetry in the magnitude of flow between the power and recovery strokes is primarily due to the faster contraction velocity of the power stroke.

\section{Phase-averaged flow measurements}

The phase-averaged flow generated by the pulsations in Cassiopea resembles a vertical blowing jet (Fig. 10A), with velocities averaged over the entire cycle of the order of $10 \mathrm{~mm} \mathrm{~s}^{-1}$ for a medusa with a bell diameter of $6 \mathrm{~cm}$. Fluid is entrained near the bell margin and ejected above the oral surface. The direction of induced motion and spreading of the jet generated by Cassiopea pulsing can be understood by an examination of the radial distribution of phase-averaged axial velocity (Fig. 11). Using the cylindrical coordinate system, the axial velocity describes the vertical or $x$-component of the velocity, and the radial velocity describes the horizontal or $r$-component. The axial velocity reaches its peak value at a distance of about 1 diameter above the tank floor. The profile peaks are centered at $r / d=-0.5$, which is the location of the medusa bell margin. Within the field of observation in these experiments, the axial velocity is reduced in magnitude upon moving from the second $(x / d=1.4)$ to the last $(x / d=1.8)$ downstream station. This is indicative of jet spreading about an axis that is centered on the bell margin, initiated by the advection of the starting vortex formed during the power stroke. The direction of induced motion is made clear by observing the axial velocities about the oral-aboral axis at $r / d=0$, herein referred to as the centerline velocity. The magnitude of the centerline velocity increases as we move downstream from the medusa, with the peak value of centerline velocity occurring at the farthest sampled axial location of $x / d=1.8$.

The phase-averaged flow generated by the numerical simulation during the fourth pulsing cycle is shown in Fig. 10B. As in the case of the experiments, fluid is entrained along the substrate and pulled into the bell and through the oral arms. In terms of the averaged flow profile out of the bell, there are significant differences between the experimental measurements and the results of the numerical simulations. In the experiments, flow is on average pulled across the oral surface, towards the central axis, and upwards away from the substrate and the organism. In the numerical simulations, the fluid that is pushed through the oral arms is pulled back into the 


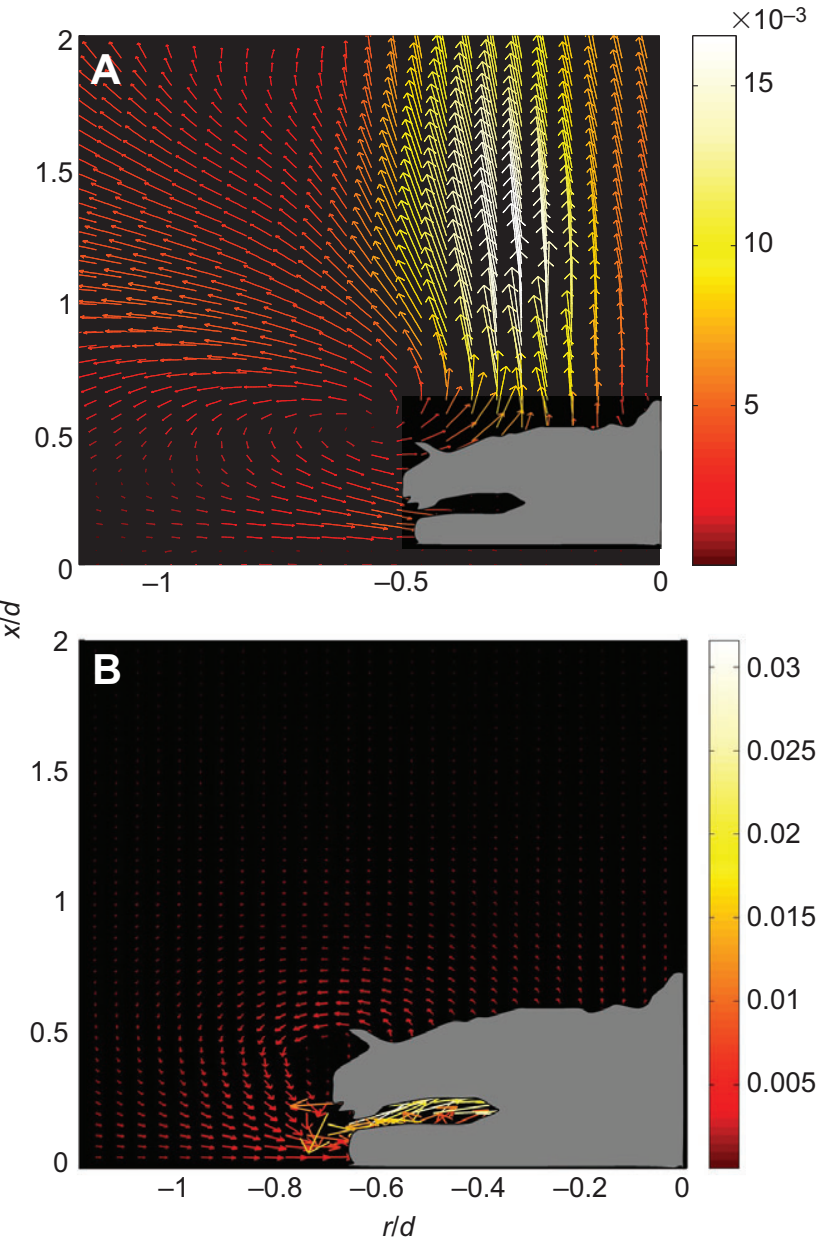

Fig. 10. Phase-averaged velocity vector field of the flow induced by a Cassiopea medusa of $6 \mathrm{~cm}$ bell diameter $(A)$ and the results of the corresponding numerical model $(B)$. The lengths of the vectors indicate their relative magnitude and their direction indicates the direction of the flow. A cartoon of the actual medusa's fully extended bell along with the outline of the oral surface is shown with the vector field. For the experimental data, the raw images were acquired at a frame rate of $30 \mathrm{~Hz}$ to provide 25-30 images per medusa pulsing cycle. Cross-correlation analysis was performed on raw image pairs separated by $0.03-0.05 \mathrm{~s}$ time intervals to obtain individual realizations. The results were averaged over 170 instantaneous velocity field realizations, which corresponds to approximately 12 pulsing cycles. For the numerical simulation, the results were averaged over 20 snapshots taken during the fourth pulsing cycle.

bell rather than being ejected upwards and away from the animal. There are a couple of possible explanations for these differences. The transport of fluid away from the animal could be a threedimensional effect caused by differences in the dynamics between stretchable vortex rings in three dimensions and straight, rigid vortex tubes in two dimensions. Other differences may be attributed to the effect of the complex geometry of the oral arms on flow. For example, the permeability of the layer could be dependent upon the direction of flow. If the oral arms are more permeable to flow moving towards the central axis than away from the central axis, the net movement of flow towards the central axis would be enhanced.

In the experiments, the spreading of the jet about the bell margin axis is accompanied by a loss in the momentum of the flow. This is compensated for by an increase in the centerline velocity with increasing distance from the medusa (Fig. 11A), in order to account for mass conservation. Thus, the direction of the induced motion

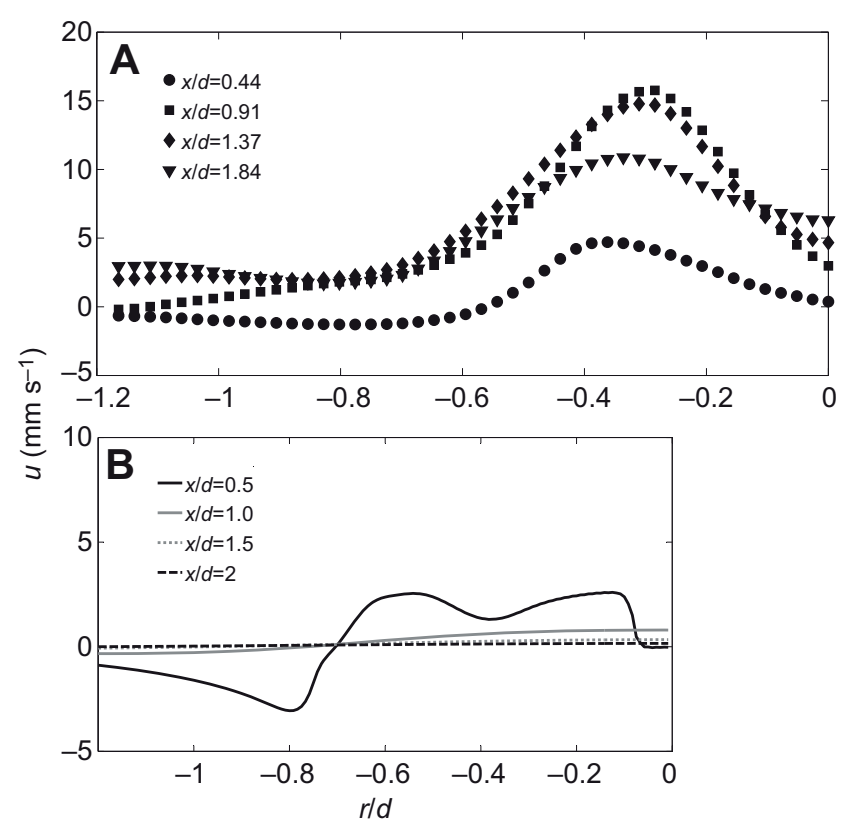

Fig. 11. Phase-averaged vertical (axial) velocity $(u)$ profiles for the flow generated by a Cassiopea medusa of $6 \mathrm{~cm}$ bell diameter $(A)$ and the corresponding numerical model (B). The velocities are shown for four dimensionless heights from the bottom of the tank $(x / d)$. The oral-aboral axis of the medusa is located at $r / d=0$, and only the left half of the flow induced by the medusa is shown.

in the flow field is toward the centerline and the direction of starting vortex advection is away from the centerline. This is similar to what is observed in Aurelia (Dabiri et al., 2005). Within the field of view used in these measurements, the peak value of axial velocity is near the bell margin where the entrainment occurs upon bell contraction. While the vertical flow velocities near the oral surface are similar for the experiments and simulations, the strong upward jet above the numerical model is not observed (Fig. 11B). Much of the flow pushed through the oral arms is instead pulled back into the bell during the subsequent pulsing cycle.

The entrainment patterns in the flow field can be examined by looking at the axial (downstream) distribution of the radial component of phase-averaged velocity (Fig. 12A). The velocity changes in sign at a distance of 0.8 diameters above the substrate. The peaks in the radial velocity values occur close to the medusa $(x / d=0.2)$, and the profiles are directed toward the centerline in a nearly horizontal manner. This pattern of flow is very similar to those generated from the numerical simulations (Fig. 12B). This suggests that the entrainment in Cassiopea occurs from the sides of the bell margin, which is different from the animal-fluid interaction of the free-swimming oblate medusae A. aurita where the fluid entrained is primarily from the upstream direction. While such horizontal entrainment is not necessarily advantageous from the standpoint of propulsive swimming, it provides a highly directed flow to the oral arms of Cassiopea. As a result, the volume of sampled water undergoes recirculation for only short periods of time before being advected into the vertical jet. As the advection of sampled water is toward the centerline, the volume of water entrained horizontally during the next power stroke is least contaminated from the previous cycle. This may allow the nonswimming medusa to effectively feed, excrete waste and exchange gas and nutrients. Flow fields for other individual medusae show similar patterns (see supplementary material Fig. S1). 

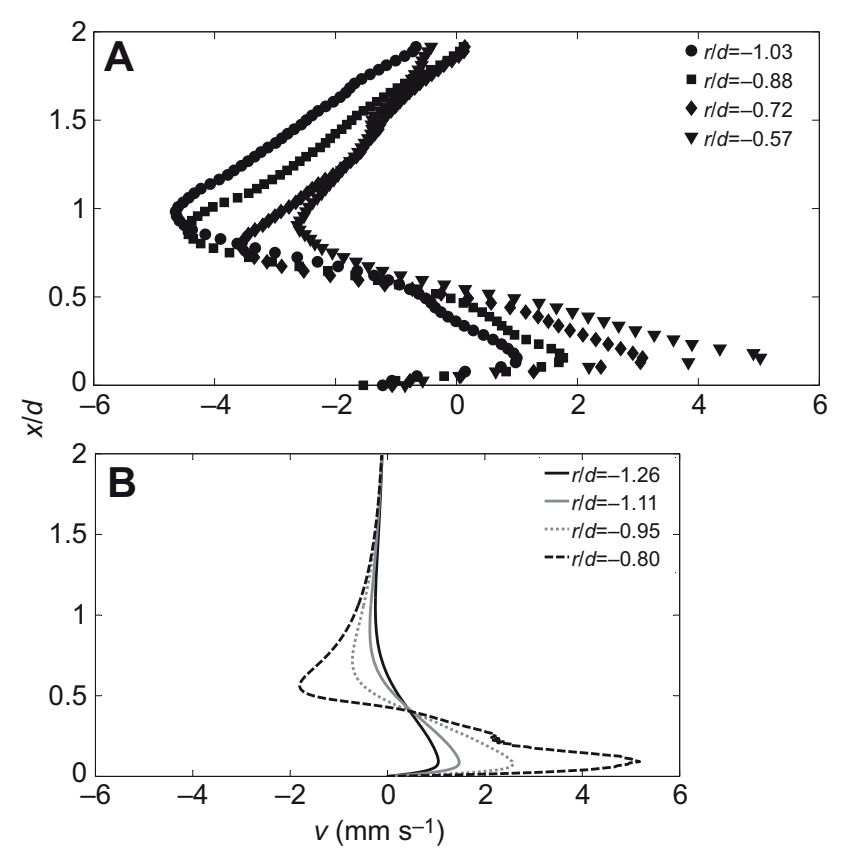

Fig. 12. Axial distribution of the radial component of phase-averaged flow velocity $(v)$ generated by a Cassiopea medusa of $6 \mathrm{~cm}$ bell diameter $(A)$ and the corresponding numerical model $(B)$. Velocities are shown for four positions of varying distance from the center of the bell $(r / d)$. These radial locations correspond to the left half of the flow field; $r / d=0$ indicates the oral-aboral axis, $r / d=-0.57$ indicates the edge of the bell margin and $r / d=-1.03$ indicates the farthest location from the medusa.

\section{DISCUSSION}

The major experimental results of this study are that (1) flow moves along the benthic substrate towards the Cassiopea medusa during the entire pulse cycle, (2) the starting vortex generated during the power stroke or contraction phase advects upward from the bell and creates strong shear flows over the oral arms, and (3) the timeaveraged flow fields show a bulk pattern of flow into the bell from along the substrate and upwards away from the organism through the oral arms. In addition, the presence of a coherent vortex wake above the oral arms is not observed. The starting and stopping vortices are broken up into more diffuse regions of vorticity that mix fluid across the entire oral arm structure. The major numerical results of this study are that (1) the simplified two-dimensional porous model does capture the flow along the substrate towards the jellyfish and through the oral arms, but (2) differences are seen between the averaged flow patterns above the oral arms measured experimentally and numerically. In particular, much of the fluid pushed through the oral arms is pulled back into the bell during the next pulsing cycle in the numerical simulations.

\section{Implications for feeding}

Vodenichar showed that carbon from zooxanthellae in most Cassiopea medusa cannot provide all of the energy necessary for basic respiratory metabolic needs (Vodenichar, 1995). Hence, both the capture of particulate nutrients and incorporation of zooxanthellae are necessary for the growth and reproduction of these organisms (Fitt and Costlety, 1998). While the specific diet of Cassiopea is not known, newly hatched nauplii of Artemia are commonly used to maintain the organisms in the lab. Fitt and Costlety recorded capture and ingestion of Artemia (Fitt and
Costlety, 1998), but details of the feeding flow patterns around the organisms have not previously been reported.

The instantaneous realizations of the flow velocities (Figs 5, 7) have clear implications for feeding in Cassiopea. Strong mixing flows are generated over the numerous secondary mouths across the oral surface. Vorticity is not centered on the bell margin but, rather, diffuses across the surface as the flow is pushed through the porous oral arms. This pattern of flow is in contrast to the local regions of mixing generated by the pelagic medusa $A$. aurita and the jet generated by the prolate jellyfish Nemopsis bachei (see Fig. 13). In the case of Aurelia, the starting vortex is centered over the tentacles that are located at the bell margin (Dabiri et al., 2005). This flow structure allows for maximum sampling and mixing of the incoming fluid at the location of the tentacles.

It is interesting to note that these differences in flow patterns correspond to the differences in morphology between the two medusae. Cassiopea spp. do not have tentacles or a central primary mouth. The eight oral arms are individually further divided into many smaller branches (7-8 pairs) (Bigelow, 1900). Contained on the distal ends of these oral arms are numerous digitata that bear nematocysts at the tips. In addition, there are club-shaped vesicles in between the arms that also bear nematocysts at the distal ends. Upon trapping prey via injecting nematocysts, the digitata bend inwards, allowing the cilia present within the oral arms to move the prey to the brachial canal that leads into the stomach for digestion. During the power stroke, the oral arms of Cassiopea move upward and the bell is contracted inward and downward such that two tasks are accomplished simultaneously: (i) the distal ends of the oral arms are brought outward and closer together in a manner that exposes the nematocyst bearing digitata to the incoming entrained flow, and (ii) the passage in between the oral arms and the vesicles that are present underneath them is widened, allowing for some fluid to collect in this subumbrellar space.

An examination of the radial component of the velocities generated during bell contraction and expansion (Fig.9A) shows that the majority of flow towards the bell is within a $1.5 \mathrm{~cm}$ region above the substrate. This observation has implications for the type of prey that Cassiopea will encounter and presumably feed upon, i.e. benthic rather than pelagic. The distribution of radial velocities generated during expansion shows that the backflow away from the bell along the substrate is isolated to a region within a couple millimeters of the bell margin (Fig.9B). The relatively small amount of backflow generated during expansion contributes to the overall large net flow pumped into the bell during the entire pulse cycle. The effect of this flow pattern is that new fluid is continually transported through the oral structures. The distribution of timeaveraged axial velocities shows that the majority of flow transported through and above the oral arms occurs in the region directly above the outer half of the medusa and tapers off as one moves towards the central axis of the organism (Fig. 11). This region of maximal flow corresponds to the location of the majority of the secondary mouths (Bigelow, 1900).

As an estimate of the encounter volume rate of fluid passing through the oral arms, the average volumetric flow rate was calculated along a vertical line drawn in Fig. 10 from $x / d=0$ to $x / d=0.5$ at radial position $r / d=-0.7$. This calculation yields a value of $30.51 \mathrm{~h}^{-1}$ of fluid brought into the bell for a $6 \mathrm{~cm}$ diameter medusa if one assumes radial symmetry in the flow field. Titelman and Hansson determined individual clearance rates of the order of $10-401 \mathrm{~h}^{-1}$ for similarly sized medusae of various pelagic species using the formula $(V / t) \times \ln \left(C_{\text {start }} / C_{\text {end }}\right)$, where $V$ is the volume of the holding tank, $t$ 


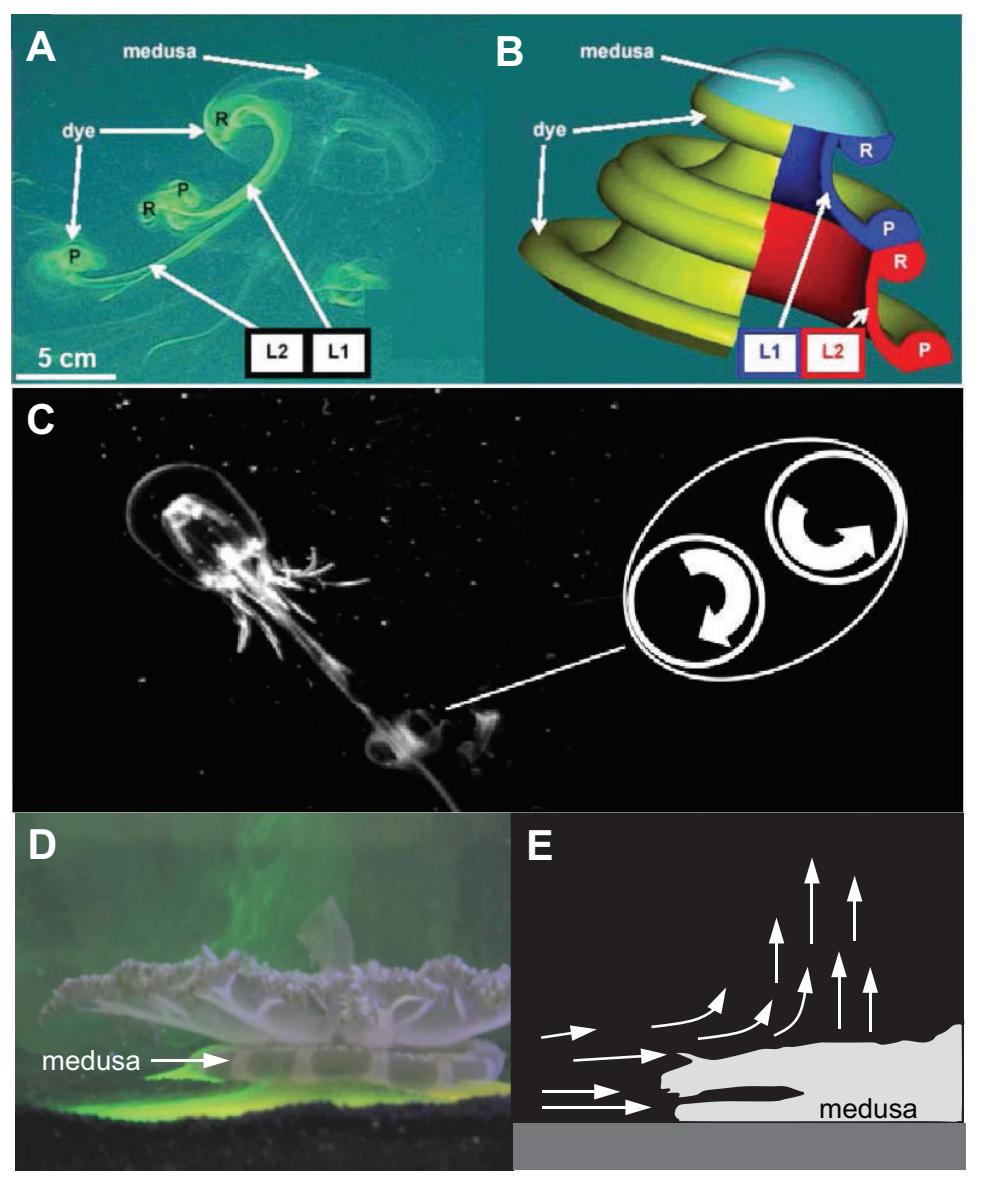

Fig. 13. (A) Flow visualization of the wake of the oblate moon jellyfish Aurelia aurita [reproduced from Dabiri et al. (Dabiri et al., 2005)]. (B) Corresponding schematic diagram of the vortex wake. P shows the vortex ring formed during contraction, $\mathrm{R}$ shows the vortex ring generated during bell expansion, and L1 and L2 indicate the adjacent lateral vortex superstructures. (C) Flow visualization of vortex formation in the prolate jellyfish Nemopsis bachei [reproduced from Dabiri et al. (Dabiri et al., 2006)]. The starting vortex generated during contraction is rapidly swept downstream of the jellyfish. (D) Flow visualization showing the wake of the upside-down jellyfish Cassiopea spp. (E) Corresponding schematic diagram. Flow is pulled into the bell from along the substrate. The starting and stopping vortices are broken up as they are pushed through the oral arms so that no coherent vortex wake is observed.

is the time elapsed, and $C_{\text {start }}$ and $C_{\text {end }}$ are the initial and final prey concentrations, respectively (Titelman and Hansson, 2006). If we assume a capture efficiency in the range of 30-80\% for Cassiopea (Acuña et al., 2011), then the clearance rate for a $6 \mathrm{~cm}$ diameter medusa would be between 9.16 and $24.431 \mathrm{~h}^{-1}$, which is within the range for other species.

\section{Cassiopea as nutrient suction pumps}

The time-averaged realizations of the velocities generated during the entire pulse cycle have implications for the flux of nutrients near the Cassiopea medusa (Fig. 10). Fluid near the substrate is pulled towards the bell and upward through the oral arms and away from the medusa. Similarly, it is likely that net flow is also generated through porous substrates, such as sand, and upwards into the water column. This effect is shown in supplementary material Movie 3 using fluorescein injected underneath the layer of sand. The addition of the medusa to the quiescent tank dramatically enhanced the advection and diffusion of dye into the water column. The observation that Cassiopea spp. can generate flow through such substrates agrees with the results of Jantzen and colleagues (Jantzen et al., 2010). They suggest that the bell pulsations of Cassiopea spp. act as suction pumps that draw nutrient-rich pore waters from permeable coral reef sediments. Through this action, Cassiopea spp. may facilitate benthic-pelagic coupling and primary production in oligotrophic coral reefs.

One question to be answered is whether the mixing currents generated by Cassiopea spp. are strong enough to significantly affect the nutrient flux in their habitats. The time-averaged flow rates obtained for a $2 \mathrm{~cm}$ diameter medusa are of the order of $1 \mathrm{~cm} \mathrm{~s}^{-1}$, but these flow rates are likely much larger in mature medusa, which may reach up to $10 \mathrm{~cm}$ in diameter. Furthermore, Cassiopea spp. are often found in larger aggregations (Sterrer, 1986) where the total flow rates produced by all individuals are greater still (Jantzen et al., 2010). Cassiopea-generated flows are likely significant in their typical habitats, such as the bottom of inshore ponds, grass beds and mangrove swamps, were maximum flow rates in the water column may be of the order of $1 \mathrm{~cm} \mathrm{~s}^{-1}$ or lower. This suggests that Cassiopea may be an 'ecosystem engineer' capable of harnessing sediment-locked nutrients.

\section{Differences between experiments and numerical simulations}

Numerical simulations modeling the Cassiopea medusa as a twodimensional (not axisymmetric) bell and the oral arms as a Brinkman porous layer did not reproduce the strong vertical jet of fluid moving upwards and away from the jellyfish. This numerical model is similar to that studied by Hamlet and colleagues (Hamlet et al., 2011), with the modification that the oral arms have finite thickness in the case studied here. The Hamlet model describes the oral arms as an infinitely thin porous layer and also does not capture the strong vertical jet above the jellyfish. The numerical flow profiles along the substrate and immediately through the oral arms are similar to those obtained experimentally, but the differences in the vertical jet may be attributed to the fact that the flow moving upwards through the layer is pulled back into the bell rather than being pushed upwards and away from the animal.

Although the current numerical model does not capture all of the features of the flow around the jellyfish, it is useful in determining what some of the important morphological features of the organism might be. The absence of the strong upwards jet in the model suggests that the structure of the oral arms might preferentially move 
fluid towards the central axis through directional differences in permeability. This effect would enhance the amount of new fluid brought into the bell during each pulse and drive more fluid from the substrate up into the water column. Future numerical work will attempt to resolve these differences by moving the simulations into three dimensions and by modeling the oral arms as a heterogeneous porous structure. An accurate model would allow better resolution of the flow in the subumbrellar cavity and through the oral arms. Such a model would also permit the exploration of a wide range of morphological and kinematic parameters on the efficiency of the pumping mechanism.

\section{CONCLUSIONS}

This study represents the first detailed description of the feeding currents generated by a single upside-down jellyfish, Cassiopea spp., in a quiescent fluid. The pulsation of a Cassiopea bell generates a jet of fluid such that fluid enters the bell from along the substrate, is pushed through the elaborate oral arms, and is then ejected upwards and into the water column. These results are in agreement with computational studies of upside-down jellyfish suggesting that the presence of the oral arms is crucial for setting up this flow structure (Hamlet et al., 2011). The structure of the flow field generated by Cassiopea spp. likely has consequences for the type of food they eat (benthic vs pelagic species) and also may significantly affect the flux of nutrients in relatively sheltered marine environments. One possible extension of this work would be to consider how the flow structure changes when the oral arms are experimentally altered. Future work on how this pattern of fluid transport is altered or enhanced in the presence of background flow and with the addition of neighboring jellyfish is also warranted.

\section{LIST OF SYMBOLS AND ABBREVIATIONS}

$\begin{array}{ll}d & \text { bell diameter } \\ \text { DPIV } & \text { digital particle image velocimetry } \\ \mathbf{f} & \text { external force } \\ F_{\mathrm{i}} & \text { instantaneous fineness ratio } \\ h & \text { height } \\ p & \text { pressure } \\ r & \text { radial/horizontal position } \\ t & \text { time } \\ \mathbf{u} & \text { velocity vector } \\ u & \text { axial velocity } \\ v & \text { radial velocity } \\ \mathbf{x} & \text { position vector in Cartesian coordinates } \\ x & \text { axial/vertical position } \\ \alpha^{2} & \text { reciprocal of hydraulic permeability } \\ \mu & \text { dynamic viscosity } \\ \rho & \text { fluid density }\end{array}$

\section{ACKNOWLEDGEMENTS}

The authors would like to thank the following people: Jack Costello for assistance with high speed DPIV; John Dabiri for advice on flow visualization; Terry Campbell, Megan Gyoreoke and Walt Douglas for their assistance in setting up and maintaining the medusae in the laboratory aquaria; Terry Rodriguez for flow visualization footage; William Kier for insightful discussions on invertebrate swimming and muscle mechanics; and Tyson Hedrick for advice on the use of the digitizing code for quantifying medusa kinematics.

\section{FUNDING}

This research was supported by Burroughs Wellcome Fund (CASI award ID no. 1005782.01) and National Science Foundation (NSF) [grant no. DMS FRG 0854961] to L.A.M. and NSF [grant no. OCE-0351398 and OCE-0623534] and Office of Naval Research [grant no. ONR N000140810654] awards to S.P.C.

\section{REFERENCES}

Acuña, J. L., López-Urrutia, Á. and Colin, S. P. (2011). Faking giants: the evolution of high prey clearance rates in jellyfishes. Science 333, 1627-1629.

Arai, M. N. (1997). A Functional Biology of Scyphozoa. London: Chapman \& Hall.

Bajcar, T., Malacic, V., Malej, A. and Sirok, B. (2009). Kinematic properties of the jellyfish Aurelia sp. Hydrobiologia 616, 279-289.

Bartleson, R. D. (2004). Interactions of seagrass beds and the water column: effects of bed size and hydrodynamics. PhD thesis, University of Maryland, College Park.

Bigelow, R. P. (1900). The anatomy and development of Cassiopeia xamachana. Boston Soc. Nat. Hist. Mem. 5, 191-236.

Brinkman, H. C. (1947). A calculation of the viscous force exerted by a flowing fluid on a dense swarm of particles. Appl. Sci. Res. Sect. A 1, 27-34.

Costello, J. H. and Colin, S. P. (1994). Morphology, fluid motion and predation by the scyphomedusa Aurelia aurita. Mar. Biol. 121, 327-334.

Costello, J. H. and Colin, S. P. (1995). Flow and feeding by swimming scyphomedusae. Mar. Biol. 124, 399-406.

Costello, J. H., Colin, S. P. and Dabiri, J. O. (2008). Medusan morphospace: phylogenetic constraints, biomechanical solutions, and ecological consequences. Invertebr. Biol. 127, 265-290.

Dabiri, J. O., Colin, S. P., Costello, J. H. and Gharib, M. (2005). Flow patterns generated by oblate medusan jellyfish: field measurements and laboratory analyses. J. Exp. Biol. 208, 1257-1265.

Dabiri, J. O., Colin, S. P. and Costello, J. H. (2006). Fast-swimming hydromedusae exploit velar kinematics to form an optimal vortex wake. J. Exp. Biol. 209, 20252033.

Dabiri, J. O., Colin, S. P. and Costello, J. H. (2007). Morphological diversity of medusan lineages is constrained by animal-fluid interactions. J. Exp. Biol. 210, 18681873.

Dabiri, J. O., Colin, S. P., Katija, K. and Costello, J. H. (2010). A wake-based correlate of swimming performance and foraging behavior in seven co-occurring jellyfish species. J. Exp. Biol. 213, 1217-1225.

Daniel, T. L. (1983). Mechanics and energetics of medusan jet propulsion. Can. J. Zool. 61, 1406-1420.

Daniel, T. L. (1984). Unsteady aspects of aquatic locomotion. Am. Zool. 24, 121-134

Fitt, W. K. and Costley, K. (1998). The role of temperature in survival of the polyp stage of the tropical rhizostome jellyfish Cassiopea xamachana. J. Exp. Marine Biol. Ecol. 222, 79-91.

Hamlet, C. L., Santanakrishnan, A. and Miller, L. A. (2011). A numerical study of the effects of bell pulsation dynamics and oral arms on the exchange currents generated by the upside-down jellyfish Cassiopea sp. J. Exp. Biol. 214, 1911-1921.

Hedrick, T. L. (2008). Software techniques for two- and three-dimensional kinematic measurements of biological and biomimetic systems. Bioinspir. Biomim. 3, 034001

Hyman, L. H. (1940). The Invertebrates: Protozoa Through Ctenophora. New York: McGraw-Hill.

Jantzen, C., Wild, C., Rasheed, M., El-Zibdah, M. and Richter, C. (2010). Enhanced pore-water nutrient fluxes by the upside-down jellyfish Cassiopea $s p$. in a Red Sea coral reef. Mar. Ecol. Prog. Ser. 411, 117-125.

Kim, Y. and Peskin, C. S. (2006). 2-D parachute simulation by the immersed boundary method. SIAM J. Sci. Comput. 28, 2294-2312.

Leiderman, K., Miller, L. A. and Fogelson, A. L. (2008). The effects of spatial inhomogeneities on flow through the endothelial surface layer. J. Theor. Biol. 252 313-325

Lipinski, D. and Mohseni, K. (2009). Flow structures and fluid transport for the hydromedusae Sarsia tubulosa and Aequorea victoria. J. Exp. Biol. 212, 2436-2447

McHenry, M. J. and Jed, J. (2003). The ontogenetic scaling of hydrodynamics and swimming performance in jellyfish (Aurelia aurita). J. Exp. Biol. 206, 4125-4137.

Peng, J. and Dabiri, J. O. (2008a). An overview of a Lagrangian method for analysis of animal wake dynamics. J. Exp. Biol. 211, 280-287.

Peng, J. and Dabiri, J. O. (2008b). The 'upstream wake' of swimming and flying animals and its correlation with propulsive efficiency. J. Exp. Biol. 211, 2669-2677.

Peng, J. and Dabiri, J. O. (2009). Transport of inertial particles by Lagrangian coherent structures: application to predator-prey interaction in jellyfish feeding. $J$. Fluid Mech. 623, 75-84.

Peskin, C. S. (2002). The immersed boundary method. Acta Numer. 11, 479-517.

Peskin, C. S. and McQueen, D. M. (1996). Fluid dynamics of the heart and its valves In Case Studies in Mathematical Modeling - Ecology, Physiology, and Cell Biology (ed. H. G. Othmer, F. R. Adler, M. A. Lewis and J. C. Dallon), pp. 309-337. Upper Saddle River, NJ: Prentice Hall.

Sahin, M., Mohseni, K. and Colin, S. P. (2009). The numerical comparison of flow patterns and propulsive performances for the hydromedusae Sarsia tubulosa and Aequorea victoria, J. Exp. Biol. 212, 2656-2667.

Sterrer, W. (1986). Marine Fauna and Flora of Bermuda. A Systematic Guide to the Identification of Marine Organisms, pp. 158-159. John Wiley \& Sons, Inc.

Stockie, J. M. (2009). Modelling and simulation of porous immersed boundaries. Comp. Struct. 87, 701-709.

Templeman, M. A. and Kingsford, M. J. (2010). Trace element accumulation in Cassiopea sp. (Scyphozoa) from urban marine environments in Australia. Mar. Environ. Res. 69, 63-72.

Vodenichar, J. S. (1995). Ecological physiology of the scyphozoan Cassiopea xamachana. MS Thesis, University of Georgia, Athens, USA.

Welsh, D. T., Dunn, R. J. K. and Meziane, T. (2009). Oxygen and nutrient dynamics of the upside down jellyfish (Cassiopea sp.) and its influence on benthic nutrient exchanges and primary production. Hydrobiologia 635, 351-362.

Wolanski, E. (1992). Hydrodynamics of mangrove swamps and their coastal waters. Hydrobiologia 247, 141-161. 